\title{
Joint Space-Time Algebraically-Interleaved Turbo- Like Coded Incoherent MIMO Systems With Optimal and Suboptimal MAP Probability Decoders
}

\author{
Ardavan Rahimian, Farhad Mehran \\ School of EECE \\ University of Birmingham \\ Birmingham B15 2TT, West Midlands, UK \\ \{rahimian \& farhad.mehran\}@ieee.org
}

\author{
Robert G. Maunder \\ School of ECS \\ University of Southampton \\ Southampton SO17 1BJ, Hampshire, UK \\ rm@ecs.soton.ac.uk
}

\begin{abstract}
The outstanding coding gains and also the practical complexities of the turbo-coded communication systems, heavily depend on the iterative soft-decision decoding algorithm used for the soft-input/-output component decoders. The goal of this paper is to address the investigation on the energy gaps of the enhanced channel coding entity of [1], when original and also approximated logarithmic-based iterative MAP algorithms are employed for the iterative near maximum-likelihood (ML) decoding. As far as the large decoding delays of the turbo and turbo-like code structures constitute a major disadvantage for these types of channel-codes, the previously introduced high-performance channel-codes of [1] are developed for the original and also approximated iterative BCJR-MAP turbo decoders to enable the higher level of selection flexibility in error-rate versus computational complexity tradeoff. We examine the required energy per bit $E_{b}$ and the noise power spectral density $N_{0}$ ratio to attain the $B E R \approx 10^{-4}-10^{-5}$ for the differentially encoded phase-shift keying (D-PSK) MIMO Rayleigh fading channels.
\end{abstract}

Index Terms-BCJR algorithm, channel coding, maximum a posteriori (MAP), maximum-likelihood (ML) decoding, MIMO, space-time code, turbo code, wireless communications.

\section{INTRODUCTION}

The exceptional error correction capability of the turbo and turbo-like codes [2-4] made them part of many today's wireless communication standards (see [2-6] of [5]) for suppressing the deleterious degradation of the system error-rate due to inherent multipath fading of wireless propagation environments. In addition in order to enhance the downlink performance without the requirements for multiple receive antennas at the terminals and also eliminate the necessity of channel state information (CSI) at the transmitting terminals, space-time codes are one of the key techniques which are robust against the certain nonideal operating conditions of propagation environments, such as the frequency dispersion, antenna correlation, and channel estimation errors [6] hence the combined schemes i.e. encoding the turbo-coded sequences in space and time, and send them over the multi-antenna link, has attracted substantial research attention in academia and industry, in order to support the evergrowing capacity demands, and to provide top-quality systems and services for the modern digital wireless communications.
As one of the major concerns of communication theory, the received bit-error probability of the designed wireless system should be minimized by well-organized use of bandwidth and also power resources, while keeping the overall complexities feasible to reduce the buffering/decoder processing delays and consequential total equipment cost and deployment expenses. Specifically, for the case of turbo code family, it is well-known that greatest hindrance to their practical utilization lay in the complexities of the decoding algorithm. Although, far simpler than other codes of the similar performance, the structure still requires a high number of operations [7]. According to Table I, since incurred complexity for implementing the minimization of bit-error probability for BCJR-maximum a posteriori (MAP) decoding [8] (and for its variants) is significantly higher than the soft-output Viterbi algorithm (SOVA) [9], the vast body of research is dedicated for finding energy gaps or coding gain gaps (CGGs) between coded systems based on the utilization of the optimal and the suboptimal BCJR-MAP decoders; Table II tabulates the available results of the major contributions and the last findings on this subject. The MAP algorithm was firstly invented for estimating the a posteriori probabilities of the states, and the transitions of a Marcov source observed in the memoryless noise. Berrou et al. invoked this algorithm for decoding the turbo codes [10].

TABLE I. COMPUTATIONAL COMPLEXITY COMPARISON BETWEEN SOVA AND ORIGINAL/APPROXIMATED BCJR-MAP DECODING ALGORITHMS FOR $(\mathrm{N}, \mathrm{K})$ CONVOLUTIONAL COMPONENT CODES WITH MEMORY $V$ [11].

\begin{tabular}{|c|c|c|c|c|}
\hline Operation & MAP & Log-MAP & Max-Log-MAP & SOVA \\
\hline Additions & $\begin{array}{l}2^{(\mathrm{k}+\mathrm{v}+1)} \\
+6\end{array}$ & $6 \times 2^{(\mathrm{k}+\mathrm{v})}+6$ & $4 \times 2^{(\mathrm{k}+\mathrm{v})}+8$ & $2^{(\mathrm{k}+\mathrm{v}+1)}+9$ \\
\hline Multiplications & $\begin{array}{c}5 \times 2^{(\mathrm{k}+\mathrm{v})} \\
+8\end{array}$ & $2^{(\mathrm{k}+\mathrm{v})}$ & $2^{(\mathrm{k}+\mathrm{v}+1)}$ & $2^{(\mathrm{k}+\mathrm{v})}$ \\
\hline Maximizations & - & $4 \times 2^{(\mathrm{v})}-2$ & $4 \times 2^{(\mathrm{v})}-2$ & - \\
\hline Lookups & - & $4 \times 2^{(\mathrm{v})}-2$ & - & - \\
\hline Exponentiations & $2^{(\mathrm{k}+\mathrm{v}+1)}$ & - & - & - \\
\hline
\end{tabular}

\section{Design of ChanNEl-Code EnTITIES}

A. Encoder Structure and Transmission

The principle of the turbo codes based on the parallel concatenation suffers from the fact that at medium-to-high SNRs, an increase in transmit power does not lead to reduction 
TABLE II. MAJOR CONTRIBUTIONS ON COMPARISON OF ENERGY PER Bit/NOISE POWER SPECTRAL DENSITY $\left(E_{b} / N_{0}\right)$ FOR MAP (AND ITS VARIANTS) DECODERS FOR TARGETED BERS $\leq 10^{-3}$

\begin{tabular}{|c|c|c|c|c|c|c|}
\hline & \multicolumn{2}{|c|}{ System Specification } & $\begin{array}{r}\text { MAP } \\
\text { (in dBs) }\end{array}$ & $\begin{array}{c}\text { Log- } \\
\text { MAP } \\
\text { (in dBss) }\end{array}$ & $\begin{array}{l}\text { Max- } \\
\text { Log- } \\
\text { MAP } \\
\text { (in dBs) } \\
\end{array}$ & $\begin{array}{l}\text { BER } \\
\quad \approx\end{array}$ \\
\hline [12] & \multicolumn{2}{|c|}{ DVB-RCS application } & $\mathbf{N} / \mathbf{A}^{1}$ & 1.4 & 1.65 & $10^{-3}$ \\
\hline [13] & \multirow{3}{*}{$\begin{array}{c}\mathrm{N} \approx 1000-\text { bit } \\
P^{2} C^{2} \text { over } \\
\text { AWGN } \\
\text { channel with } \\
\text { BPSK }\end{array}$} & $\|_{\omega} \tau_{\omega}$ & 1.825 & 1.81 & 2 & $10^{-5}$ \\
\hline [14] & & $\omega \pi$ & 1.75 & 1.75 & 1.93 & $10^{-4}$ \\
\hline [15] & & $\omega \pi$ & N/A & 1.95 & 2.05 & $10^{-4}$ \\
\hline \multirow[b]{2}{*}{ [16] } & \multirow[b]{2}{*}{$\begin{array}{c}\text { UMTS } \\
\text { application }\end{array}$} & 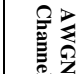 & N/A & 0.417 & 0.819 & $10^{-5}$ \\
\hline & & 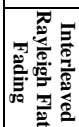 & N/A & 0.417 & 1.533 & $10^{-5}$ \\
\hline \multirow{2}{*}{ [17] } & \multirow{2}{*}{$\begin{array}{c}\text { 3GPP } \\
\text { application }\end{array}$} & 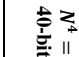 & 4.37 & N/A & 4.45 & $10^{-5}$ \\
\hline & & 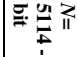 & 1.62 & N/A & 2.18 & $10^{-5}$ \\
\hline [18] & \multirow{2}{*}{$\begin{array}{c}\text { OCDMA } \\
\text { networking }\end{array}$} & 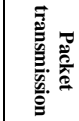 & $K_{s}^{5}=90$ & N/A & $\underset{100}{K_{s}=}$ & $10^{-5}$ \\
\hline [19] & & 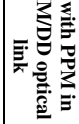 & 12.5 & N/A & 15 & $10^{-5}$ \\
\hline \multirow[t]{2}{*}{ [20] } & \multirow{2}{*}{$\begin{array}{c}\text { Duo-binary } \\
\text { Turbo Code } \\
\text { used in Wi- } \\
\text { MAX } \\
\text { standard }\end{array}$} & 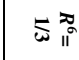 & N/A & 0.9 & 1.25 & $10^{-5}$ \\
\hline & & 志元 & N/A & 3.37 & 3.5 & $10^{-5}$ \\
\hline \multirow[t]{2}{*}{ [21] } & \multirow{2}{*}{$\begin{array}{c}\text { Rate }^{1 / 2} \\
N=1024-b i t \\
\text { PCC }(\text { FF } / \\
\left.\text { FB }^{7}\right)= \\
(17,15)_{\text {oct }}\end{array}$} & $\begin{array}{l}\stackrel{8}{8} \\
\frac{\pi}{\pi}\end{array}$ & 1.9 & N/A & 2.1 & $10^{-4}$ \\
\hline & & $\begin{array}{l}f \\
0 \\
0 \\
3\end{array}$ & 8.95 & 8.95 & 9.35 & $10^{-4}$ \\
\hline
\end{tabular}

${ }^{1} \mathrm{~N} / \mathrm{A}$ : not computed in this investigation

${ }^{2}$ PCC: parallel concatenated codes.

${ }^{3} \mathrm{~L}$ : component code's constraint length

${ }^{4} \mathrm{~N}$ : interleaver size.

${ }^{5} \mathrm{~K}_{\mathrm{s}}$ : average number of photons per symbol.

${ }^{6} \mathrm{R}$ : code rate

${ }^{7} \mathrm{FF} / \mathrm{FB}$ : feedforward/feedback.

of bit-errors. Therefore, the turbo coding community dedicated a vast body of research for raising the minimum distance of the code; hence, improving the flare performance. One way to increase the minimum distance is by configuring the same ingredients of turbo codes (i.e. component encoders/decoders and interleaver/deinterleaver) in serial concatenation fashion as proposed in [22]. The serial concatenation scheme has shown to yield the high-performance comparable, and in some cases, superior to the parallel concatenation schemes [3].

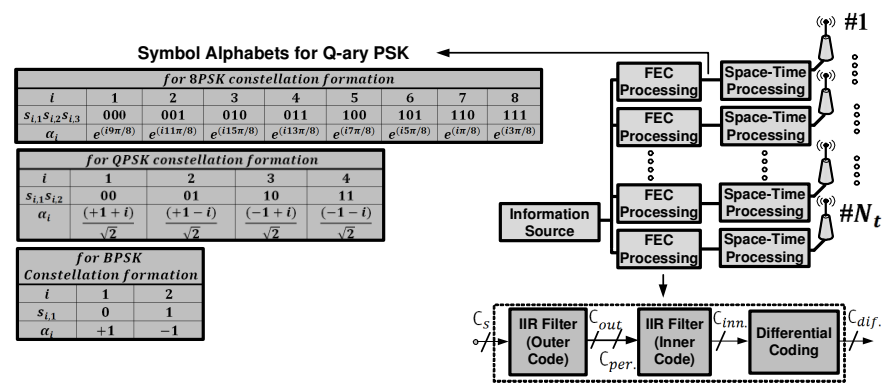

Figure 1. Bit-level turbo-like encoder with DPSK and space-time coding.

The encoder of interest is shown in Fig. 1. It is composed of two cascaded digital IIR (infinite impulse response) filters. The 4-state outer convolutional component codec with the rate $R_{0}=$ $1 / 2$, and the inner convolutional component codec with the rate $R_{i}=2 / 4$, with generating matrices given by:

$G_{(D)}^{\text {out }}=\left[1 \frac{1+D^{2}}{1+D+D^{2}}\right]$

$G_{(D)}^{\text {inner }}=\left[1 \frac{1+D^{2}}{1+D+D^{2}} \frac{D+D^{2}}{1+D+D^{2}} 0 ; 01 \frac{1+D^{2}}{1+D+D^{2}} \frac{D+D^{2}}{1+D+D^{2}}\right]$

The $C_{s}, C_{\text {out }}, C_{\text {per }}, C_{\text {inn. }}$, and $C_{\text {dif. }}$ denote the $\left(\frac{N_{I N T}}{2}\right)$ - bit source sequence (i.e. a sequence $\left\{m_{i}\right\}$ of binary message where $0 \leq$ $i \leq\left(\frac{N_{I N T}}{2}\right)-1$ and $\left.m_{i} \in\{0,1\}\right), N_{I N T}-$ bit outer coded sequence, $N_{I N T}$-bit permuted sequence, $2 N_{I N T}$-bit inner coded sequence (i.e. coded bits $\left\{x_{v}\right\}, 0 \leq v \leq\left(\frac{N_{I N T}}{2 R_{\text {overal }}}\right)-1$ ), and $2 N_{I N T}-$ bit differentially coded baseband sequence. Together with the constituent codecs, it is mainly the permutor that determines the distance spectrum and the minimum distance of the overall code. That is one of the major issues of theoretical and practical turbo code design since it essentially determines the error-rate performance of the code. In particular, the performance of short-length turbo codes is highly determined by the permutation structure [23]. Permutation is a process of interleaving the order of a data sequence in a one-to-one deterministic format in order to create statistical independence between disturbances on parity symbols related to the same information symbol entering the channel encoders [24]. Many permutation algorithms have been investigated to improve the distance properties of the code while maintain the simple and practical hardware implementations. The most used interleaver type in communication systems is block interleaver [25], which has been extensively investigated and employed in turbo coded systems. For the spreading factor of permutation function $\pi$, one way is to express in terms of the displacement vector $(\Delta \alpha, \Delta \beta) \in Z^{2}$ of a pair of the input values $i<\bar{i}$, a given permutation has spreading factor $\left(s_{1}, s_{2}\right)$ if wherever $\Delta \alpha<s_{1}$, then $|\Delta \beta| \geq s_{2}$ [26]. To study the randomness of the permutor, the set of displacement vectors of a block interleaver can be used [26]: $\quad D_{l}=\left\{(\Delta \alpha, \Delta \beta) \in Z^{2} \mid \Delta \alpha=\omega-x, \Delta \beta=\pi(\omega)-\right.$ $\left.\pi(x), 0 \leq x<\omega<N_{I N T}+1\right\}$; the largest set of the displacement vectors could be attained for Costas arrays [26-27] which are square arrays of dots/1s and blanks/0s such that: a) there exists exactly one dot/1 in each row and column; b) no four dots $/ 1 \mathrm{~s}$ form a parallelogram; and c) no tree dots/1s lying on a straight line are equidistant [28]; i.e. let $n \in N^{*}$ and $A$ be a permutation matrix, if $a_{i j}=1$, set $f(j)=i$, with $i, j \in\{1,2, \ldots, n\}$; we call $A$ a Costas array of dimension $n$ iff [29]: 
$\forall i_{1}, i_{2}, i_{3}, i_{4} \in\{1,2, \ldots, n\}, i_{1} \leq i_{2}, i_{3} \leq i_{4}:\left(i_{1}-i_{2}, f\left(i_{1}\right)-\right.$

$\left.f\left(i_{2}\right)\right)=\left(i_{3}-i_{4}, f\left(i_{3}\right)-f\left(i_{4}\right)\right) \rightarrow i_{1}=i_{3}, i_{2}=i_{4}$.

Costas arrays appeared for the first time in connection with describing a novel frequency hopping pattern for the Sonar systems and have been an active topic of Radar/Sonar research [30-31]. They have also been applied in the other areas of the communications engineering; such as [32]. There is a website which provides up-to-dated technical reports and publications on Costas arrays [33].

In order to investigate the fundamental Costas permutation mapping, let us consider a bijection $f:[n] \rightarrow[n] ; f$ is a Costas array iff [34-35]: let $[n]:=\{1, \ldots, n\}, n \in N^{N_{I N T}, \forall i, j, k \text { such that }}$ $1 \leq i, j, i+k, j+k \leq n: f(i+k)-f(i)=f(j+k)-f(j) \rightarrow i=$ $j$ or $k=0$. Based on the Welch's algebraic construction [28,36], and according to [37], let $p$ be a prime and $\vartheta$ be a primitive root of the finite field $F(p)$ of $p$ elements, and let $d \in[p-1]-1$ be a constant then the function $f:[p-1] \rightarrow[p-1]$ where $f(i)=$ $\vartheta^{i-1+c} \bmod p$ is a Costas permutation of order $p-1$; hence the output of permutor is the bit-level randomized outer-coded bit sequence, and is inserted into the inner codec. The functionality of permutor is described by invertible permutation function $\pi$ on the numbers $\left\{0,1, \ldots, N_{I N T}\right\}$ :

$\pi:\left\{0,1, \ldots N_{I N T}\right\} \rightarrow\left\{0,1, \ldots N_{I N T}\right\}$

The fundamental permutation [38] $\pi$ is given by (5) as:

$\pi=\left(\begin{array}{cccc}0 & 1 & \cdots & N_{I N T}-1 \\ \pi(0) & \pi(1) & \cdots & \pi\left(N_{I N T}-1\right)\end{array}\right)$

Its inverse permutation is denoted by $\pi^{-1}$, which maps the permuted sequence back to the original order i.e. we have $\pi^{-1}(\pi(i))=i$ with:

$\pi=\left(\begin{array}{cccc}0 & 1 & \cdots & N_{I N T}-1 \\ \pi^{-1}(0) & \pi^{-1}(1) & \cdots & \pi^{-1}\left(N_{I N T}-1\right)\end{array}\right)$

Therefore, as an instance, the fundamental Costas interleaver arrays for permutation size $N_{I N T}=18$ are given by:

$\pi^{2}=$

$\left(\begin{array}{llllllllllllllllll}1 & 2 & 3 & 4 & 5 & 6 & 7 & 8 & 9 & 10 & 11 & 12 & 13 & 14 & 15 & 16 & 17 & 18\end{array}\right)$

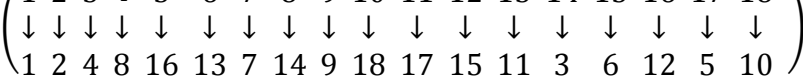

$\pi^{3}=$

$\left(\begin{array}{llllllllllllllllll}1 & 2 & 3 & 4 & 5 & 6 & 7 & 8 & 9 & 10 & 11 & 12 & 13 & 14 & 15 & 16 & 17 & 18\end{array}\right)$

$\left(\begin{array}{cccccccccccccccccc}\downarrow & \downarrow & \downarrow & \downarrow & \downarrow & \downarrow & \downarrow & \downarrow & \downarrow & \downarrow & \downarrow & \downarrow & \downarrow & \downarrow & \downarrow & \downarrow & \downarrow & \downarrow \\ 1 & 3 & 9 & 8 & 5 & 15 & 7 & 2 & 6 & 18 & 16 & 10 & 11 & 14 & 4 & 12 & 17 & 13\end{array}\right)$

$\pi^{13}=$

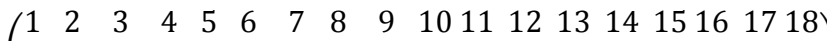

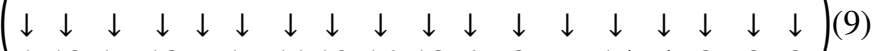

$\left(\begin{array}{llllllllllllllllll}1 & 13 & 17 & 12 & 4 & 14 & 11 & 10 & 16 & 18 & 6 & 2 & 7 & 15 & 5 & 8 & 9 & 3\end{array}\right)$

where, the index is the primitive element of $\mathrm{GF}(19)$. Before forming constellations from the $C_{i n n}$, the differential coding (DC) is performed by taking the difference between the present information bit and the past encoded bit; by taking advantage of the high level of correlation between neighboring samples in the data sequence by the encoding differences; hence, at time $k$,
$C_{\text {dif. } k}=C_{\text {inn. } k} \oplus C_{\text {dif. } k-1}(\oplus$ stands for the modulo-2 addition $)$. In digital wireless communications, there are a great number of scenarios over fading channels in which the transmission has to be performed without having reliable carrier phase estimation at the reception side. Hence, phase ambiguity is likely to be present in the received signal due to imperfect knowledge of the carrier's phase and fading effects. These phase errors can be pre-compensated by $\mathrm{DC}$ at the transmitter and incoherent detection at the receiver [39-40]. DC is computationally-simple technique widely used to enable non-coherent detection, when the carrier phase acquisition and the tracking are impossible /unreliable, or prohibitively complex due to successive phase noise, commonly happened in cases where noise and errors of local oscillators are considerable [41]. This is a great advantage when the waveform is passed through many circuits in a communication system and network changes (e.g. switching between data paths), result in occasional change in constructive sense of the output [42]. Moreover, that is well recognized for protecting bit sequence against unintentional polarity reversals [43]. Hence they are useful in many bad channel conditions e.g. for mobile users at cell boundary who experience bad qualityof-service (QoS), and for estimating channel gains significant amount of training data is required [44]. Although they result in deterioration of coding gain for $\approx 2 d B$ since whenever an error occurs on a single bit in the received data sequence that tends to propagate as double error, their robustness in the fading environments, and also their easier implementations made them widely used technique in the digital communication systems.

The signal constellations are formed from $C_{\text {dif }}$. by using phase-shift keying (PSK) i.e. $I_{n}=e^{j \theta_{n}}$. Since the modulation is differential, at each $k^{\text {th }}$ transmission interval, the data phasor $\varphi_{k}=\varphi_{k-1}+\Delta \varphi_{k}$ is sent, where $\Delta \varphi_{k} \in\{0,2 \pi / Q, \ldots, 2 \pi(Q-1) / Q\}$ is determined by the sequence of $\log _{2}^{Q}$ input bits using the Gray and Binary mapping rule [45-46]. The transmission sequences for space-time encoding for twin-antenna and triplet-antenna systems are given in (32) and (37) of [47]. The Rayleigh-faded MIMO wireless channel with the PSK input is described by:

$y=H x+n$

where, $H \in C^{N_{r} \times N_{t}}$, and

$x \in X=$

$\left\{\left(x_{1} x_{2} \cdots x_{N_{t}}\right)^{T} \mid x_{N_{t}}=\frac{\exp \left\{\frac{j 2 \pi q}{Q}\right\}}{\sqrt{N_{t}}}, 1 \leq m \leq N_{t}, 0 \leq q \leq Q-1\right\}$

where, $y, n \in C^{N_{r} \times 1}, Q$ is the number of points in the PSK constellation, and $N_{t}$ and $N_{r}$ are the number of transmit and receive antennas, respectively (superscript $T$ denotes transpose) [48]. The definition of $X$ essentially enforces the $Q$-ary PSK input constraint and also implies uniform power allocation at the transmitter; the values $x$ can take guarantee that the average energy from each transmit antenna is $1 / N_{t}$ and elements of $H$ are i.i.d. complex Gaussian $\sim N_{c}(0,1)$ [47]. Those of noise $n$ are i.i.d. complex Gaussian $\sim N_{c}\left(0, \sigma^{2}\right)$, where $\sigma^{2}=1 / \gamma_{s}[48]$ and $\gamma_{s}$ is the symbol SNR $E_{s} / N_{0}[48]$.

\section{B. Reception and Channel Decoder}

At reception side, the decision criteria expressed in (17)(20) of [49] are used for detecting symbols of the system with 
two transmitting antennas; meanwhile the maximum-likelihood (ML) detector amounts to minimize the decision metrics given in appendix of [50] for detecting symbols of the system with three transmitting antennas. Then, the detected symbols are differentially decoded (i.e. $\tilde{\mathrm{C}}_{k}=\tilde{\mathrm{C}}_{r . k} \oplus \tilde{\mathrm{C}}_{r . k-1}$, where $\tilde{\mathrm{C}}_{k}$ stands for the decisions taken on the transmitted information bits and $\tilde{\mathrm{C}}_{r . k}$ presents the received bits), and demodulated; and then the demodulated signals are fed into the iterative turbo decoder. The turbo decoder consists of the soft-input/-output constituent decoders for corresponding convolutional codecs, linked by an interleaver and a deinterleaver (depicted in Fig. 2). Owing to the presence of permutor, optimum maximum-likelihood (ML) decoding algorithms for the turbo codes are very complex. However, their iterative decoding in which soft information is exchanged between sub-blocks of decoder is quite feasible and leads to near capacity performance. This information could be regarded as a type of diversity in that it can refine constituent decoder's outputs in each iteration [19]. A single decoding is performed in each iteration using the channel observations and reliability information delivered by other component decoder that has acted before [19]. This iterative process can be applied to the BCJR-MAP decoding invoked by Berrou et al. for decoding turbo codes, and also to SOVA algorithm advocated in [51-53]. A fundamental difference between the BCJR-MAP and SOVA algorithms for decoding turbo coded sequences is that while the former minimizes the probability of bit-error, the latter minimizes the probability of sequence-error which yields considerable performance penalty but with the lower amount of the computational complexities. In SOVA, in order to find the most probable information sequence, only two paths in trellis

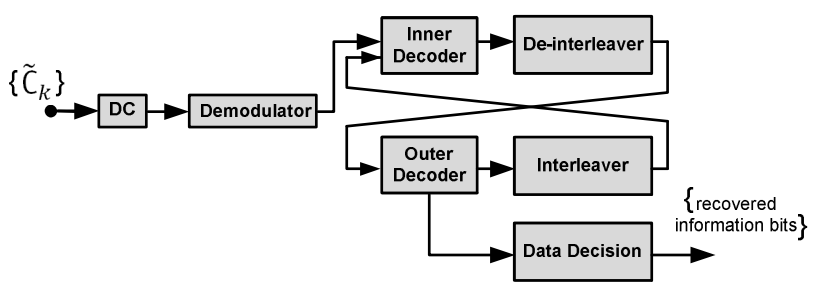

(a)

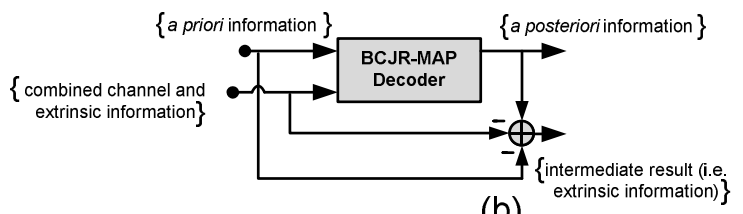

(b)

Figure 2. (a) Bit-level turbo-like differential decoder; (b) soft-input/-output component decoder.

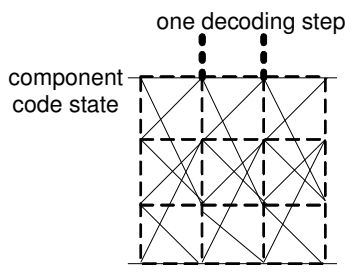

(a)

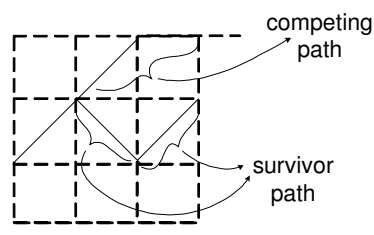

(b)
Figure 3. (a) MAP uses all the paths in the trellis in order to optimally determine the reliability of the bit; (b) SOVA takes two paths in order to determine the most probable information sequence. diagram are considered but the competing path may not be the best competing path. As shown in Fig. 3, only one competing path at every decoding step is computed, in each decoding iteration; compared to the SOVA, where the sequence error probability is minimized, in the MAP, all the paths in the trellis diagram are considered at each decoding step; therefore, in order to approach the capacity performance, the computation complexity grows significantly. The soft-input and soft-output constituent decoders put out not only the various bits, but also the confidence it has in a specific decision which is described by the Log-Likelihood Ratio (LLR). The LLR is the ratio of the a posteriori probability (APP) of each information bit value. Suppose $u, y, \pi(\omega)$, and $工($.$) are denoting the information$ sequence, noise-corrupted version of codeword corresponding to $u$, permuted version of $\omega$, and the map from the information bits to parity check bits. For codeword $x=(u, \beth(u), \beth(\pi(u)))$ corresponding to the information sequence $u$, the decoder must compute the LLR for each data bit $u_{k}$, which is given by $L_{k}=$ $P\left(u_{k}=1 \mid y\right) / P\left(u_{k}=0 \mid y\right)$, for $k=1,2, \cdots, N_{I N T}$. If $L_{k} \geq 1$, the decoder estimates that $u_{k}$ is equal to one, otherwise, it is then estimated to be equal to zero. Hence the turbo decoding process can be regarded as the iterative improvement of the APPs since the LLR is the ratio of the APPs of each information bit value. The APP is given by the equation (12) as [53]:

$L\left(u_{k}\right)=\log \frac{\sum_{\overline{\bar{S}}_{k}} \sum_{\overline{\bar{S}}_{k-1}} \gamma_{1}\left(C_{k}\right) \cdot \alpha_{k-1}\left(\overline{\bar{S}}_{k-1}\right) \cdot \beta_{k}\left(\overline{\bar{S}}_{k}\right)}{\sum_{\overline{\bar{S}}_{k}} \sum_{\bar{S}_{k-1}} \sum_{i=0}^{1} \gamma_{1}\left(C_{k}\right) \cdot \alpha_{k-1}\left(\overline{\bar{S}}_{k-1}\right) \cdot \beta_{k}\left(\overline{\bar{S}}_{k}\right)}$

where, $\alpha_{k}(),. \beta_{k}($.$) , and \gamma_{k}($.$) are the forward, backward, and$ branch transition parameters, respectively; given by:

$\alpha_{k}\left(\overline{\bar{S}}_{k}\right)=\frac{\sum_{\overline{\bar{S}}_{k}} \sum_{i=0}^{1} \gamma_{i}\left(C_{k}\right) \cdot \alpha_{k-1}\left(\overline{\bar{S}}_{k-1}\right)}{\sum_{\overline{\bar{S}}_{k}} \sum_{\bar{S}_{k-1}} \sum_{i=0}^{1} \gamma_{i}\left(C_{k}\right) \cdot \alpha_{k-1}\left(\overline{\bar{S}}_{k-1}\right)}$

and,

$\beta_{k}\left(\overline{\bar{S}}_{k}\right)=\frac{\sum_{\overline{\bar{S}}_{k+1}} \sum_{i=0}^{1} \gamma_{i}\left(\mathrm{C}_{k+1}\right) \cdot \beta_{k+1}\left(\overline{\bar{S}}_{k+1}\right)}{\sum_{\bar{S}_{k}} \sum_{\bar{S}_{k+1}} \sum_{i=0}^{1} \gamma_{i}\left(C_{k+1}\right) \cdot \alpha_{k}\left(\overline{\bar{S}}_{k}\right)}$

and,

$$
\begin{gathered}
\gamma_{i}\left(C_{k}\right)=\operatorname{Pr}\left(y_{k}^{S} \mid u_{k}=i\right) \cdot \operatorname{Pr}\left(y_{k}^{p} \mid u_{k}=i, \overline{\bar{S}}_{k}=m, \overline{\bar{S}}_{k-1}=\hat{\vartheta}\right) \\
\operatorname{Pr}\left(u_{k}=i \mid \overline{\bar{S}}_{k}=\vartheta, \overline{\bar{S}}_{k-1}=\hat{\vartheta}\right) \cdot \operatorname{Pr}\left(\overline{\bar{S}}_{k}=m \mid \overline{\bar{S}}_{k-1}=\hat{\vartheta}\right)
\end{gathered}
$$

where, $\overline{\bar{S}}_{k}$ is the state of the encoder at time $k,(\vartheta, \vartheta) \in \aleph_{k}^{i}$ with $\aleph_{k}^{i}$ the set of transitions $\overline{\bar{S}}_{k-1}=\vartheta \rightarrow \overline{\bar{S}}_{k}=\vartheta$ that are due to $u_{k}=i$, and $y_{k}^{S}$ and $y_{k}^{p}$ are noise corrupted channel outputs for $d_{k}$ and parity bits at time $k$ respectively, and $C_{k}$ denotes the collection $\left(y_{k}^{S}, y_{k}^{p}, \overline{\bar{S}}_{k-1}, \overline{\bar{S}}_{k}\right)$. Hence, evaluation of APP could be summarized as the algorithm presented in Fig. 4. As far as the MAP algorithm is very complex, due to the amount of the computations, it could be then simplified by performing the operations in the logarithmic domain [54-56]. The forward, backward, and transition metrics could be calculated in the logarithmic domain using the Jacobian logarithm [14]. Using this approach, the multiplication in equation (13) and (14) is replaced by the addition in order to save the computations: $\hat{a}_{k}(s)=\max \left[\hat{a}_{k-1}(\hat{s})+\gamma_{k}(\widehat{s}, s)\right]$ and $\hat{\beta}_{k-1}(\hat{s})=\max \left[\hat{\beta}_{k}(s)+\right.$ $\left.\gamma_{k}(\widehat{s}, s)\right]$; where, $\hat{a}_{k}(s), \hat{\beta}_{k}(s)$, and $\hat{\gamma}_{k}(\widehat{s}, s)$ are logarithmic values of the forward, backward, and transition metrics, respectively. 


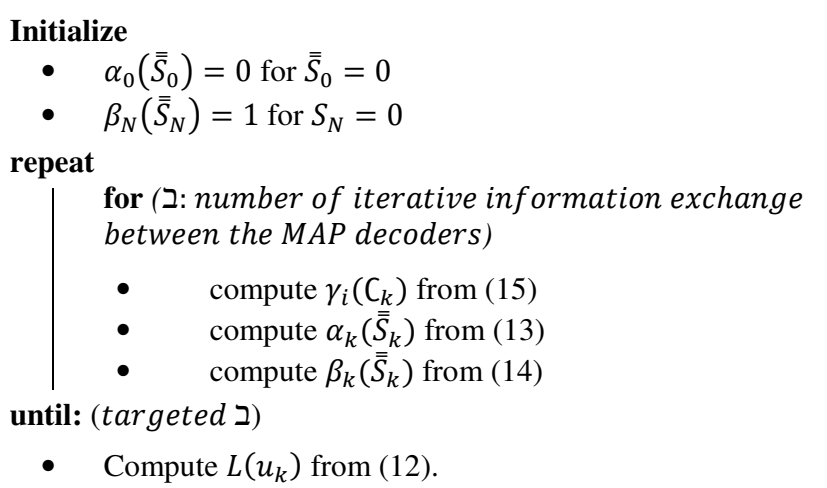

Figure 4. Summary of the algorithmic evaluation of APP.

In order to preserve the original MAP algorithm and yield equivalent performance, all maximizations can be augmented over the two values with the correction function. The Jacobian logarithm has been computed as: $\max ^{*}(x, y)=\max \left(x_{1}, x_{2}\right)+$ $\log \left(1+e^{-\left|x_{1}-x_{2}\right|}\right)=\max \left(x_{1}, x_{2}\right)+f_{c}(|y-x|)$; i.e. maximum of the function's two arguments plus a nonlinear correction function, that is only a function of the absolute difference between the two arguments; i.e. $f_{c}(|y-x|)$ depicted in Fig. 5. Thus, the extrinsic information bit in the equation (12) can be derived in terms of the new metrics [14] as:

$L\left(u_{k}\right)=\max _{((\hat{s}, s), u=+1)}\left[\hat{a}_{k-1}(\hat{s})+\hat{\gamma}_{k}(\widehat{s}, s)+\hat{\beta}_{k}(s)\right]-$
$\max _{((\hat{s}, s), u=-1)}\left[\hat{a}_{k-1}(\hat{s})+\hat{\gamma}_{k}(\widehat{s}, s)+\hat{\beta}_{k}\right]$

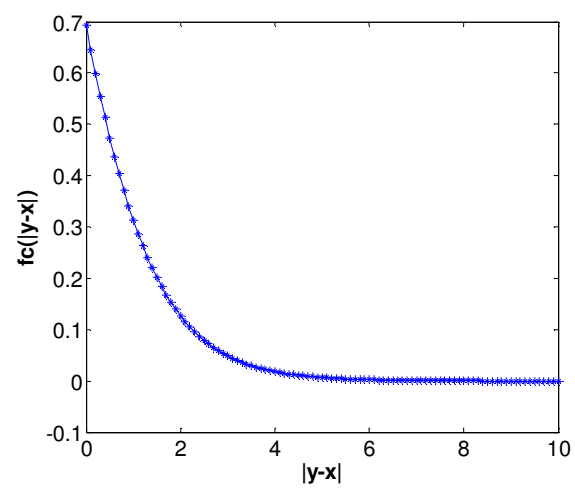

Figure 5. The correction function.

The significant computations saving also arise since division operation is replaced by the subtraction in logarithmic domain. This algorithm which tries to preserve the original MAP by calculating the bit-error probability with less the computation complexity is known as Log-MAP algorithm. If we avoid using the correction function term to further reduce the amount of the complexity, the resultant decoding algorithm is known as MaxLog-MAP algorithm [55], which is only based on the selection and comparison operations; the Jacobian logarithm is loosely approximated using: $\max ^{*}(x, y) \approx \max \left(x_{1}, x_{2}\right)$. Typically, for more than two input values, the Jacobian logarithm is applied recursively; e.g. considering three values yields: $\max ^{*}(x, y, z) \approx$ $\max ^{*}\left\{\max ^{*}(x, y), z\right\}$. The Max-Log-MAP which consists of the two Viterbi algorithms (one running in the forward and another in the backward direction [56]) is the least complex of the true MAP and Log-MAP, (note that it has twice the complexity of the SOVA for each half-decoding iteration) but offers the worst
BER performance [16]. The Log-MAP avoids approximation in the Max-Log-MAP algorithm and presents the performance equivalent to the original MAP, while does not manifest the major disadvantage of the true MAP (i.e. high computational complexity). The correction only requires an additional onedimensional lookup table (LUT), and also an addition per max operation [57]. The relationship among the MAP, Log-MAP, Max-Log-MAP, and SOVA, has been depicted in Fig. 6.

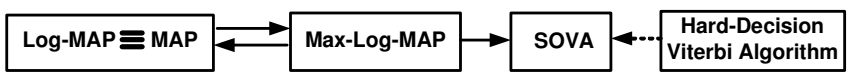

Figure 6. Relationship among: MAP, Log-MAP, Max-Log-MAP, and SOVA [57].

\section{ERROR-RATE PERFORMANCE EVALUATIONS: ALGORITHM AND NUMERICAL RESULTS}

Based on the above mentioned discussions, in this section, we present the computer simulations that compute the required energies per bit $E_{b}$ over noise power spectral density $N_{0}$ ratio $\left(E_{b} / N_{0}\right)$ to attain BER $\approx 10^{-4}$ and $10^{-5}$ over MIMO Rayleigh fading channels (tabulated in Table III and IV). The Algorithm 1 presents a simplified algorithm of the actual code used in the conducted performance evaluations for MAP and its simplified versions. For the transmission over multiple-antenna links, the concatenation of the space-time codes for twin-/triplet-antenna systems [49-50] are used after DC and forming gray-coded and binary-coded PSK constellations (denoted by the GC and BC, respectively). For predicting the probability density function (PDF) of entries of channel matrix in which each element is the channel impulse response between the $j^{\text {th }}$ transmit antenna and the $i^{\text {th }}$ receive antenna, Rayleigh distribution which is the most commonly used model for describing statistical time-varying nature of received signal's envelope in the urban environments with the blocked line-of-sight (LoS), has been utilized.

As it is presented, performance of iterative turbo receiver utilizing maximization operation with the correction function $f_{c}(|y-x|)($ Log-MAP algorithm) preserves the performance of the MAP decoded system; i.e. the energy gaps fall below 0.2 $d B$; hence, when one considers high-performance (near ML) iterative decoding algorithm for the application of recovering encoded bit sequences of aforementioned channel codes, it is suggested to employ the Jacobian logarithm [14] for calculating the forward, backward, and transition metrics in the logarithmic domain hence we can conclude that for the introduced channelcodes the required $\mathrm{SNR}$ in order to yield $\mathrm{BER} \approx 10^{-4}-10^{-5}$ remain marginally the same, like what happens in the PCCcoded systems; such as: [13-14] and [21].

Taking into account computational complexity comparisons given in Table I, the considerable computational saving could be achieved while also reducing dynamic range problems when calculations of APPs are performed solely based on applying the addition, multiplication, and maximization operations; i.e. omitting $f_{c}(|y-x|)$ in computing $\max ^{*}(x, y)$. According to the [58], taking into account the addition, subtraction, are multiplication, division, and comparison operations as the one equivalent addition per operation; taking into account the maximization and minimizations as the two equivalent additions per operation, and consider LUT as three numbers of 


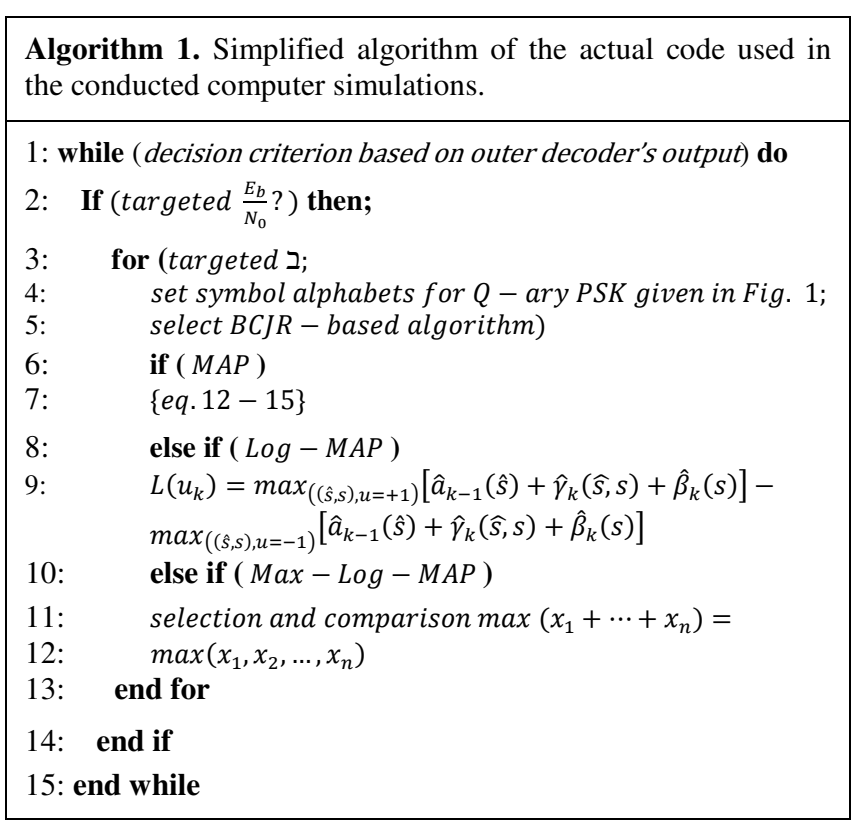

TABLE III. THE REQUIRED $E_{b} / N_{0}$ TO ACHIEVE BER $=10^{-4}$ FOR $N=600$ (AND PRIMITIVE ELEMENT 373).

\begin{tabular}{|c|c|c|c|c|c|c|c|c|c|c|}
\hline$N_{t}$ & 2 & \multicolumn{2}{|c|}{2} & \multicolumn{2}{|c|}{2} & 3 & \multicolumn{3}{|c|}{3} & \\
\hline$N_{r}$ & 2 & \multicolumn{2}{|c|}{2} & \multicolumn{2}{|c|}{2} & 3 & \multicolumn{2}{|c|}{3} & \multicolumn{2}{|c|}{3} \\
\hline \multirow[b]{2}{*}{$M$} & \multirow[b]{2}{*}{2} & \multicolumn{2}{|c|}{4} & \multicolumn{2}{|c|}{8} & & \multicolumn{2}{|c|}{4} & \multicolumn{2}{|c|}{8} \\
\hline & & $B C$ & $G C$ & $B C$ & $G C$ & 2 & $B C$ & $G C$ & $B C$ & $\overline{G C}$ \\
\hline MAP & $\begin{array}{l}6.7 \\
\mathrm{~dB}\end{array}$ & $\begin{array}{c}10.9 \\
\mathrm{~dB}\end{array}$ & $\begin{array}{l}9.7 \\
\mathrm{~dB}\end{array}$ & $\begin{array}{c}16.3 \\
\mathrm{~dB}\end{array}$ & $\begin{array}{c}13.6 \\
\mathrm{~dB}\end{array}$ & $\begin{array}{l}3.5 \\
\mathrm{~dB}\end{array}$ & $\begin{array}{l}7.6 \\
\mathrm{~dB}\end{array}$ & $\begin{array}{c}6 \\
\mathrm{~dB}\end{array}$ & $\begin{array}{c}13.1 \\
\mathrm{~dB}\end{array}$ & $\begin{array}{l}9.9 \\
\mathrm{~dB}\end{array}$ \\
\hline $\begin{array}{l}\text { Log- } \\
\text { MAP }\end{array}$ & $\begin{array}{l}6.8 \\
\mathrm{~dB}\end{array}$ & $\begin{array}{c}11.1 \\
\mathrm{~dB}\end{array}$ & $\begin{array}{l}9.8 \\
\mathrm{~dB}\end{array}$ & $\begin{array}{c}16.4 \\
\mathrm{~dB}\end{array}$ & $\begin{array}{c}13.6 \\
\mathrm{~dB}\end{array}$ & $\begin{array}{l}3.5 \\
\mathrm{~dB}\end{array}$ & $\begin{array}{l}7.6 \\
\mathrm{~dB}\end{array}$ & $\begin{array}{l}6.1 \\
\mathrm{~dB}\end{array}$ & $\begin{array}{c}13.2 \\
\mathrm{~dB} \\
\end{array}$ & $\begin{array}{l}10 \\
\mathrm{~dB} \\
\end{array}$ \\
\hline $\begin{array}{l}\text { Max- } \\
\text { Log- } \\
\text { MAP }\end{array}$ & $\begin{array}{c}7 \\
\mathrm{~dB}\end{array}$ & $\begin{array}{c}11.6 \\
d B\end{array}$ & $\begin{array}{l}10.1 \\
\mathrm{~dB}\end{array}$ & $\begin{array}{l}16.8 \\
\mathrm{~dB}\end{array}$ & $\begin{array}{l}14 \\
\mathrm{~dB}\end{array}$ & $\begin{array}{l}4.1 \\
\mathrm{~dB}\end{array}$ & $\begin{array}{l}7.9 \\
\mathrm{~dB}\end{array}$ & $\begin{array}{l}6.5 \\
\mathrm{~dB}\end{array}$ & $\begin{array}{l}13.5 \\
\mathrm{~dB}\end{array}$ & $\begin{array}{c}10.3 \\
\mathrm{~dB}\end{array}$ \\
\hline
\end{tabular}

TABLE IV. THE REQUIRED $E_{b} / N_{0}$ TO ACHIEVE BER $=10^{-5} \mathrm{FOR}$ $N=600$ (AND PRIMITIVE ELEMENT 373).

\begin{tabular}{|c|c|c|c|c|c|c|c|c|c|c|}
\hline$N_{t}$ & 2 & \multicolumn{2}{|c|}{2} & \multicolumn{2}{|c|}{2} & 3 & \multicolumn{2}{|c|}{3} & \multicolumn{2}{|c|}{3} \\
\hline$N_{r}$ & 2 & \multicolumn{2}{|c|}{2} & \multicolumn{2}{|c|}{2} & 3 & \multicolumn{2}{|c|}{3} & \multicolumn{2}{|c|}{3} \\
\hline \multirow[b]{2}{*}{$M$} & \multirow[b]{2}{*}{2} & \multicolumn{2}{|c|}{4} & \multicolumn{2}{|c|}{8} & & \multicolumn{2}{|c|}{4} & \multicolumn{2}{|c|}{8} \\
\hline & & $B C$ & $G C$ & $B C$ & $\overline{G C}$ & 2 & $\overline{B C}$ & $\overline{G C}$ & $B C$ & $G C$ \\
\hline MAP & $\begin{array}{l}8.8 \\
\mathrm{~dB}\end{array}$ & $\begin{array}{l}13 \\
\mathrm{~dB}\end{array}$ & $\begin{array}{c}11.7 \\
\mathrm{~dB}\end{array}$ & $\begin{array}{c}18.3 \\
\mathrm{~dB}\end{array}$ & $\begin{array}{c}15.7 \\
d B\end{array}$ & $\begin{array}{l}5.6 \\
d B\end{array}$ & $\begin{array}{l}9.5 \\
\mathrm{~dB}\end{array}$ & $\begin{array}{l}8.1 \\
\mathrm{~dB}\end{array}$ & $\begin{array}{l}15 \\
d B\end{array}$ & $\begin{array}{l}12 \\
\mathrm{~dB}\end{array}$ \\
\hline $\begin{array}{l}\text { Log- } \\
\text { MAP }\end{array}$ & $\begin{array}{l}8.8 \\
d B\end{array}$ & $\begin{array}{c}3.1 \\
\mathrm{~dB}\end{array}$ & $\begin{array}{c}11.7 \\
\mathrm{~dB}\end{array}$ & $\begin{array}{c}18.3 \\
\mathrm{~dB}\end{array}$ & $\begin{array}{c}15.7 \\
d B\end{array}$ & $\begin{array}{l}5.6 \\
d B\end{array}$ & $\begin{array}{l}9.5 \\
\mathrm{~dB}\end{array}$ & $\begin{array}{l}8.1 \\
\mathrm{~dB}\end{array}$ & $\begin{array}{l}15 \\
\mathrm{~dB}\end{array}$ & $\begin{array}{c}2.1 \\
\mathrm{~dB}\end{array}$ \\
\hline $\begin{array}{l}\text { Max- } \\
\text { Log- } \\
\text { MAP }\end{array}$ & $\begin{array}{c}9 \\
\mathrm{~dB}\end{array}$ & $\begin{array}{c}13.5 \\
\mathrm{~dB}\end{array}$ & $\begin{array}{c}11.9 \\
\mathrm{~dB}\end{array}$ & $\begin{array}{c}18.7 \\
\mathrm{~dB}\end{array}$ & $\begin{array}{l}16 \\
d B\end{array}$ & $\begin{array}{c}6 \\
\mathrm{~dB}\end{array}$ & $\begin{array}{l}9.8 \\
\mathrm{~dB}\end{array}$ & $\begin{array}{l}8.5 \\
\mathrm{~dB}\end{array}$ & $\begin{array}{c}15.2 \\
d B\end{array}$ & $\begin{array}{c}12.4 \\
\mathrm{~dB}\end{array}$ \\
\hline
\end{tabular}

equivalent additions, the overall complexities of Log-MAP and Max-Log-MAP algorithms are $\left(48 \cdot 2^{v}-13\right)$ and $\left(28 \cdot 2^{v}-3\right)$, respectively (expressed in terms of the number of equivalent additions), where $v$ is the memory order of the system encoder; meanwhile, as our numerical results suggest, the deterioration of coding gain falls below $\approx 0.5 d B$; hence, this slightly higher $E_{b} / N_{0}$ requirements for yielding the same error-rate translates into a considerable decrease in the complexity and sensitivity. Hence, the obtained results recommend that for the discussed channel-code entity, the iterative turbo decoders utilize MaxLog-MAP algorithm are good compromises between system performance and complexity.

In addition, for the case of $Q=4$ - and 8-PSK constellation formations with the gray mapping where the labels of adjacent symbols differ only in one bit; i.e. $\{00,01,11,10\}$ and $\{000,001,011,010,110,111,101,100\}$ for $Q=\{4,8\}$, respectively; additional coding gains of up to 1.5 and $3 d B$ attained. Hence, the results confirm to use then gray mapping rather than the binary-coded bit assignment; i.e. $\{00,01,10,11\}$ and $\{000,001,010,011,111,100,101,110,111\}$ for $Q=\{4,8\}$ in order to form signal constellations; which yields additional coding gains without incurring additional difficulties.

Moreover it is worth mentioning here that we defer to make comparisons for cases that the Monte Carlo simulation results may seem to be useless and/or the result of the investigations is predictable based on available literature for generic turbo coded system model with of 90-99\% confidence level; the two major ones have been discussed briefly as follows:

(1) Comparison with block interleavers: block interleaver is the most used interleaver type in communication systems [25]. In [1], the authors already suggest that serial turbo-like codes employing the Welch-constructed Costas interleavers of length 100-bit and 600-bit result in additional coding gains of up to 3 and $4 d B$ for attaining the BER $\approx 10^{-5}$ and $10^{-6}$, respectively; in case of $3 \times 4$ MIMO wireless transmissions with the coherent BPSK digital modulation.

(2) Investigation for different interleaver sizes: It may seem to be required to characterize the performance of generic serial turbo-like codes for AWGN and fading channels for varying interleaver lengths since in design of conventional serial turbolike codes [3], Benedetto et al. introduced that large coding gains of up to $2 d B$ could be achieved by increasing the interleaver size of 200-bit to 2000-bit (at the expense of further buffering delays which is equal to $N_{I N T} \cdot f_{b}$ where $f_{b}$ is the bit rate [59]). In response to this potential idea of investigation, careful attention must be paid into the interleaver lengths to be selected in wireless systems. Modern wireless applications tend to be short-frame (less than 1000-bit). As the original work on serial turbo-like codes and also many other works on this class of channel-codes suggest, the system performance predictions and resultant coding gains for the in-range interleavers lengths (i.e. $100 \leq N_{I N T} \leq 1000$ ) remain the same; hence, the important interleaver length range is already investigated and available on literature since 100-bit and 600-bit codes of [1] are the good representative lengths; higher sizes (i.e. $1000 \ll N_{I N T}$, and in some conventional cases, on the orders of thousands bits; e.g. Berrou's classic PCCs with $N_{I N T}=65536$ ) are not of particular interest in modern digital communications applications due to the resultant long delays.

\section{CONCLUSSION AND PERSPECTIVES}

In an attempt to find the efficient and high-performance (in terms of the error-rate and overall complexity) algorithms to be employed in designing the permutor and unscramblers of serial turbo-like codes (which are of particular interest due to better 
performance in flare region rather than classic schemes), in [1], it has been shown that space-time turbo-like codes employing the Welch-constructed Costas interleavers with representative $N_{I N T} \leq 1000$ lengths result in the coding gains of $1-2 d B$ for attaining $\mathrm{BER}<10^{-3}$ for $2 \times 2$ Rayleigh-faded MIMO systems, and $2-5 d B$ for achieving BER $<10^{-5}$ for $3 \times 4$ Rayleigh-faded MIMO systems, when they are compared with the same system model based on the block interleavers of $10 \times 10$ and $24 \times 25$ dimensions. Hence, as in many other related key investigations (i.e. listed in Table II), a great deal of interest which motivated us in order to study the coding gains associated with exploiting optimal and suboptimal MAP decoding algorithms in spacetime turbo-like codes of [1], to demonstrate that to what extend we can save energy while tolerating the higher complexities. The numerical results and comparisons have been all extended for $Q$-ary PSK with gray and binary bit assignments, over $2 \times 2$ and 3x3 MIMO wireless systems (enjoying the STBCs [47, 49, 50], in conjunction with designed channel codes). Meanwhile, during this comprehensive investigation, we further detailed the potential analysis concerned with design of the channel encoder /decoder of [1], by presenting fundamental permutation arrays for the Welch-constructed Costas interleavers.

The results of this article can be extended in a number of ways; the following outlined issues are of particular interest:

(1) As we pointed out briefly, the delay of turbo processing is considered as the main bottleneck of their application and utilization in the modern hard and firm real-time wireless data transmissions [60]. Hence finding strategies for preserving their performance while keeping the complexities lower are still important open research issues concentrated by the information theory community; In [15] it is suggested to use the Maclaurin series expansion instead of the logarithmic term in the Jacobian logarithmic function in order to enable direct implementation of Jacobian logarithmic function by comparators and adders, as this is the case for Max-Log-MAP algorithm. The outstanding high-performance very close to Log-MAP decoding while also outperforming the Max-Log-MAP algorithm in the waterfall region of the BER performance curves are presented in [15], when the communication channel is AWGN. Developing this decoding algorithm for channel-codes presented in this article over SISO/MIMO fading channels is of particular theoretical and practical importance.

(2) In [61], Wang et al. proposed two new matrix transform algorithms for Max-Log-MAP decoding of parallel turbo codes which considerably speed up the decoding operations and also lowers the computational system complexities which facilitates the implementation of the Max-Log-MAP algorithm in specialpurpose VLSI hardware for the parallel processing. Hence, this algorithm could be extended in the case of Log-MAP and also Talakoub et al. decoder [15], and employ the resultant iterative turbo decoder for the space-time turbo-like codes presented in this investigation.

(3) As in the case of [1], in this article, we have assumed transmitters are uninformed about the channel state information (CSI). As far as the CSI affects the capacity of MIMO systems significantly, developing the detailed performance analysis and evaluations for the case of discussed channel codes employed in conjunction with the MIMO technologies with the available CSI at the $\mathrm{Tx}(\mathrm{s})$ is an important open issue.

(4) Analog circuits may need lower power and the area compared with digital implementations in high-speed iterative applications; hence, in [62] design and test results for a fullyintegrated translinear tail-biting MAP decoder with a goal of verifying the feasibility of the CMOS translinear circuits for the MAP decoders implementation is developed. Developments for the specific turbo codes (as in our scheme) may result in the revolutionary changes from key practical design aspects, i.e. power consumption and occupied space.

(5) In order to take advantage of the trade-offs between the BER, code rate, spectral efficiency, and decoder complexity; the introduced FECs have potential advantage of enjoying the Khandani et al.'s turbo coding method [63], which parses the message frame into $n$-bit symbols and permutes on a symbolby-symbol basis.

(6) In [64], the turbo-coded OFDM transmissions have been analyzed; developing the combination of SCQICs with clipping is of particular interest in order to reduce the peak-to-average power ratio (PAPR), and achieve the provisioning performance with the reasonable redundancy.

\section{REFERENCES}

[1] F. Mehran and R. G. Maunder, "Wireless MIMO systems employing joint turbo-like STBC codes with bit-level algebraically-interleaved URSCs," IEEE Int. Wireless Symp. (IWS), China, Apr. 2013.

[2] C. Berrou, A. Glavieux, and P. Thitimajshima, "Near Shannon limit error-correcting coding and decoding: Turbo codes," IEEE ICC, Jun. 1993, pp. 1064-1070.

[3] S. Benedetto, D. Divsalar, G. Montorsi, and F. Pollara, "Serial concatenation of interleaved codes: Performance analysis, design, and iterative decoding," IEEE Trans. Inf. Theory, vol. 44, pp. 909-926, May 1998.

[4] D. Divsalar, H. Jin, and R. J. McEliece, "Coding theorems for "turbolike" codes," 36th Allerton Conf. Commun., Control, and Comput., Allerton, IL, Sep. 1998, pp. 201-210.

[5] F. Mehran and A. Rahimian, "Physical layer performance enhancement for femtocell SISO/MISO soft real-time wireless communication systems employing serial concatenation of quadratic interleaved codes," $20^{\text {th }}$ Iranian Conf. Elect. Eng. (ICEE), May 2012, pp. 1188-1193.

[6] A. Naguib, V. Tarokh, N. Seshadri, and A. R. Calderbank, "A spacetime coding modem for high-data-rate wireless communications," IEEE J. Sel. Areas Commun., vol. 16, no. 8, pp. 1459-1477, Oct. 1998.

[7] C. P. Dennett, "An investigation of turbo codes over mobile wireless channels," PhD Dissert., University of Wolverhampton, 2006.

[8] L. R. Bahl, J. Cocke, F. Jelinek, and J. Raviv, "Optimal decoding of linear codes for minimizing symbol error rate," IEEE Trans. Inf. Theory, vol. 22, pp. 284-287, Mar. 1974.

[9] G. Ungerboeck, "Channel coding with multilevel/phase signals," IEEE Trans. Inf. Theory, vol. IT-28, no. 1, pp. 55-67, Jan. 1982.

[10] C. Berrou and A. Glavieux, "Near optimum error correcting coding and decdoing: Turbo codes," IEEE Trans. Commun., vol. 44, no. 10, pp. 1261-1271, 1996.

[11] B. Vucetic and J. Yuan, "Turbo codes: principles and applications," Kluwer Academic Publishers, 2000.

[12] M. C. Valenti, S. Cheng, and R. I. Seshadri, "Turbo and LDPC codes for digital video broadcasting," Ch. 12, Turbo Code Applications: A Journey from a Paper to Realization, Springer, 2005.

[13] L. Hanzo and B. Y. T. H. Liew, Turbo Coding, Turbo Equalisation and Space-Time Coding. New York: Wiley-IEEE Press, 2002. 
[14] P. Robertson, E. Villebrun, and P. Hoeher, "A comparison of optimal and sub-optimal MAP decoding algorithms operating in the log domain," IEEE ICC, 1995, pp. 1009-1013.

[15] S. Talakoub and B. Shahrrava, "A linear Log-MAP algorithm for turbo decoding over AWGN channels," Proc. Electro/Inform. Technol. Conf., Milwaukee, WI, Aug. 2004.

[16] M. C. Valenti and J. Sun, "The UMTS turbo code and an efficient decoder implementation suitable for software defined radios," Int. J. Wireless Inf. Netw., vol. 8, no. 4, pp. 203-216, Oct. 2001.

[17] J. Vogt, and A. Finger, "Improving the max-log-MAP turbo decoder," Electron. Lett., vol. 36, pp. 1937-1939, 2000.

[18] J. Y. Kim and H. V. Poor, "Turbo-coded optical direct-detection CDMA system with PPM modulation," J. Lightw. Technol., vol. 19, no. 3, pp. 312-322, Mar. 2001.

[19] J. Y. Kim and H. V. Poor, "Turbo-coded packet transmission for an optical CDMA network," J. Lightw. Technol., vol. 18, no. 12, pp. 19051916, Dec. 2000.

[20] S. Papaharalabos, et al., "Non-recursive max* operator with reduced implementation complexity for turbo decoding," IET Commun., vol. 6, no. 7, pp. 702-707, 2012.

[21] H. R. Sajadpour, "Maximum a posteriori decoding algorithms for turbo codes," Proc. SPIE, vol. 4045, pp. 73-83, Jul. 2000.

[22] S. Benedetto and G. Montorsi, "Serial concatenation of block and convolutional codes," Electron. Lett., vol. 32, pp. 887-888, 1996.

[23] K. Enokizono and H. Ochiai, "A simple interleaver design for variablelength turbo code," $6^{\text {th }}$ Int. Symp. Turbo Codes and Iterative Inf. Process. (ISTC), Sep. 2010, pp. 354-358.

[24] A. Wakeel, D. Kronmueller, W. Henkel, H. B. Neto, "Leaking interleavers for UEP turbo codes," $6^{\text {th }}$ Int. Symp. on Turbo Codes and Iterative Inf. Process. (ISTC), Sep. 2010, pp. 191-195.

[25] F.-H. Huang, "Evaluation of soft output decoding for turbo codes," Master Thesis, Virginia Tech, 1997.

[26] C. Heegard and S. B. Wicker, Turbo Coding, Kluwer Academic Publishers, 1999.

[27] S. W. Golomb and H. Taylor, "Constructions and properties of Costas arrays," Proc. of the IEEE, vol. 72, no. 9, pp. 1143-1163, Sep. 1984

[28] K. Drakakis, F. Iorio, and S. Rickard, "The enumeration of Costas arrays of order 28," IEEE Inf. Theory Workshop (ITW), Sep. 2010, pp. 1-5.

[29] K. Drakakis, "Reduced complexity symbol detectors with parallel structures for ISI channels," Turkish J. Elec. Eng. and Comput. Sciences, vol. 15, no. 1, pp. 67-76, Mar. 2007.

[30] S. Haykin, Communication Systems, $5^{\text {th }}$ Ed. New York: Wiley, 2009.

[31] J. P. Costas, "Medium constraints on Sonar design and performance," GE Co., Tech. Rep. Class 1 Rep. R65EMH33, 1965.

[32] H. Koorapaty et al., "Pilot design for a cellular wireless system based on Costas arrays," IEEE PIMRC, Sep. 2010.

[33] Available online: http://www.costasarrays.org.

[34] K. Drakakis, "On the hops present in Costas permutations," IEEE Trans. Inf. Theory, vol. 56, no. 3, pp. 1271-1277, Mar. 2010.

[35] K. Drakakis, "A structural constraint for Golomb Costas arrays," IEEE Trans. Inf. Theory, vol. 56, no. 11, pp. 5762-5764, Nov. 2010.

[36] S. W. Golomb and G. Gong, "The status of Costas arrays," IEEE Trans. Inf. Theory, vol. 53, no. 11, pp. 4260-4265, Nov. 2007.

[37] K. Drakakis, et al. "Distance vectors in Costas arrays," $42^{\text {th }}$ Annl. Conf. Inf. Sciences and Syst., Mar. 2008, pp. 1234-1239.

[38] A. Huebner and R. Jordan, "On higher order permutors for serially concatenated convolutional codes," IEEE Trans. Inf. Theory, vol. 52, no. 3, pp. 1238-1248, Mar. 2006.

[39] F. Gini and G. B. Gianna, "Generalized differential encoding: a nonlinear signal processing framework," First IEEE Workshop on Signal Process. Advances in Wireless Commun., 1997, pp. 153-156.

[40] R. F. H. Fischer, et al., "Coded modulation for differential encoding and non-coherent reception on fading channels," Int. ITG Conf. on Systems, Commun., and Coding (SCC), Jan. 2000, pp. 139-146.
[41] M. Peleg, et al., "On interleaved, differentially encoded convolutional codes," IEEE Trans. Inf. Theory, vol.45, no.7, pp. 2572-2582, Nov. 1999.

[42] "Line codes," Available online: http://www.engineer.tamuk.edu/cleung/EEEN4329/9_Line\%20Code.pdf

[43] C. Langton, "Tutorial 2- differential encoding," Available online: http://www.complextoreal.com/tutorials.

[44] M. R. Bhatnagar and A. Hjorungnes, "Differential coding for MAC based two-user MIMO communication systems," IEEE Wireless Commun., vol. 11, no. 1, pp. 9-14, Jan. 2012.

[45] I. B. Djordjevic and B. Vasic, "Multilevel coding in $M$-ary DPSK/Differential QAM high-speed optical transmission with direct detection," J. Lightw. Technol., vol. 24, no. 1, pp. 420-428, Jan. 2006.

[46] J. G. Proakis, Digital Communications, MA: McGraw-Hill, 2001.

[47] V. Tarokh, et al., "Space-time block codes from orthogonal designs," IEEE Trans. Inf. Theory, vol. 45, pp. 1456-1467, Jul. 1999.

[48] W. He and C. N. Georghiades, "Computing the capacity of a MIMO fading channel under PSK signaling," IEEE Trans. Inf. Theory, vol. 51, no. 5, pp. 1794-1803, May 2005.

[49] S. M. Alamouti, "A simple transmit diversity technique for wireless communications," IEEE J. Sel. Areas Commun., vol. 16, no. 8, pp. 1451-1458, Oct. 1998.

[50] V. Tarokh, H. Jafarkhani, and A. Calderbank, "Space-time block coding for wireless communications: Performance results," IEEE J. Sel. Areas Commun., vol. 17, pp. 451-460, Mar. 1999.

[51] J. Hagenauer and P. Hoeher, "A Viterbi algorithm with soft-decision outputs and its applications," IEEE Global Telecommun. Conf., 1989, pp. 47.1.1-47.1.7.

[52] J. Hagenauer, "Source-controlled channel decoding," IEEE Trans. Commun., vol. 43, pp. 2449-2457, Sep. 1995.

[53] W. Gu, et al., "Turbo code decoding in MAI environment," $6^{\text {th }}$ Int. Symp. Turbo Codes and Iterative Inf. Process. (ISTC), Sep. 2010, pp. 206-210.

[54] W. Koch and A. Baier, "Optimum and sub-optimum detection of coded data distributed by time-varying inter-symbol interference," IEEE Global Telecommun. Conf., Dec. 1990, pp. 1679-1684.

[55] J. Erfanian, S. Pasupathy, and G. Gulak, "Reduced complexity symbol detectors with parallel structures for ISI channels," IEEE Trans. Commun., vol. 42, pp. 1661-1671, Feb./Mar./Apr. 1994.

[56] M. C. Valenti, "Iterative detection and decoding for wireless communications," PhD Dissert., Virginia Tech, 1999.

[57] P. Robertson, et al., "Optimal and sub-optimal maximum a posteriori algorithms suitable for turbo decoding," European Trans. Telecommun., vol. 8, no. 2, pp. 119-125, Mar./Apr. 1997.

[58] I. A. Chatzigeorgiou, et al.,"A comparison of convolutional and turbo coding schemes for broadband FWA systems", IEEE Trans. Broadcast., vol. 53, no.2, pp. 494-503, Jun. 2007.

[59] M. Rice, P. Gray, and A. Barbulescu, "Coding and modulation techniques for high speed data services by satellite" Proc. ICICS, vol. 1, pp 435-438, 1997.

[60] F. Mehran, "Interleaver choice in serially concatenated codes for soft real-time wireless speech transmission applications," $19^{\text {th }}$ Iranian Conf. Elect. Eng. (ICEE), May 2011.

[61] D. Wang and H. Kobayashi, "Matrix approach for fast implementations of logarithmic MAP decoding of turbo codes," IEEE Pacific Rim Conf. Commun., Comput., and Signal Processing (PACRIM), Aug. 2001, vol. 1, pp. 115-118

[62] C. Winstead, et al., "CMOS analog MAP decoder for $(8,4)$ hamming code," IEEE J. Solid-State Circuits, vol. 39, no. 1, pp. 122-133, Jan. 2004.

[63] M. Bingeman and A. K. Khandani, "Symbol-based turbo codes," IEEE Commun. Lett., vol. 3, no. 10, pp. 285-287, Oct. 1999.

[64] H. Nikopour, et al., "Turbo-coded OFDM transmission over a nonlinear channel," IEEE Trans. Veh. Technol., vol. 54, no. 4, pp. 1361-1371, Jul. 2005 . 